kurz wirksame Nitrate (3,3 vs. 0,6 Spraystöße oder Kapseln pro Woche). Erwartungsgemäß sank auch die Herzfrequenz, und zwar von 82,9 auf 70,4 pro Minute. 97\% der Ärzte beurteilten die Wirksamkeit als sehr gut oder gut.

\section{Effekt zusätzlich zur Betablockade}

Auch die Kombination mit Betablockern (Metoprolol, Bisoprolol, Carvedilol, Nebivolol) wurde untersucht. Die Subgruppenanalyse $(n=344)$ ergab, dass die Patienten von Ivabradin profitierten, auch wenn sie gleichzeitig einen Betablocker anwendeten. Der If-Kanal-Hemmer führte zu einer zusätzlichen Reduktion der Herzfrequenz, die vergleichbar hoch ausfiel wie im Gesamtkollektiv. Die Häufigkeit von Anginapectoris-Anfällen nahm um $79 \%$ ab.

Bestätigt wurde auch die Verträglichkeit des $\mathrm{I}_{\mathrm{f}}$-Kanal-Hemmers. Im viermonatigen Beobachtungszeitraum wurden nur 78 unerwünschte Arzneimittelwirkungen beobachtet, vor allem Übelkeit und Schwindel.

- Dr. Judith Neumaier

Quellen: Köster R et al. Am Heart J. 2009;

Ebelt $\mathrm{H}$ et al. Clin Res Cardiol. 2009;

Presseinformation von Servier

DGP-Leitlinie zu akuter Bronchitis

\title{
Starke Empfehlung für Pflanzenkombination
}

- In der Leitlinie der Deutschen Gesellschaft für Pneumologie und Beatmungsmedizin (DGP) zur "Diagnostik und Therapie von erwachsenen Personen mit akutem und chronischem Husten" werden Sekretolytika zurückhaltend beurteilt: „Es gibt derzeit wenig methodisch einwandfreie Studien zur Bewertung der Wirksamkeit in Hinblick auf die Linderung des akuten Hustens", heißt es dort. Einzige Ausnahme ist das pflanzliche Kombinationspräparat Bronchipret ${ }^{\oplus}$. Für das Phytotherapeutikum wird bei „,mittelgradiger Evidenz" eine "starke Empfehlung" ausgesprochen.

Grundlage für die Empfehlung sind zwei randomisierte kontrollierte Studien, die placebokontrolliert und doppelblind durchgeführt wurden. Bronchipret ${ }^{\oplus}$ wurde in einer Studie als Saft mit Wirkstoffen aus Thymian und Efeu, in der anderen Studie in Form von Filmtabletten mit Wirkstoffen aus Thymian und Primel gegeben.

An den Studien beteiligten sich 363 bzw. 362 Patienten mit akuter Bronchitis mit produktivem Husten und erschwertem Abhusten. Die Zahl der Hustenanfälle, der primäre Studienendpunkt, war an den Tagen 7-9 mit dem Thymian-Efeu-Saft um $68,7 \%$ (Placebo 47,6\%) zurückgegangen, mit den Thymian-Primel-Tabletten um 67,1\% (Placebo 51,3\%). Mit beiden Darreichungen konnte die Zahl der Hustenanfälle zwei Tage früher halbiert werden als mit Placebo. Die Verträglichkeit der Pflanzenextrakt-Kombination wurde von mehr als 95\% der Prüfärzte sowie der Patienten als gut oder sehr gut eingestuft.

$\mathrm{Zu}$ vergleichbaren Ergebnissen kommt eine prospektive Anwendungsbeobachtung mit dem Thymian-Efeu-Saft bei 1234 Kindern und Jugendlichen ab zwei Jahren. Die Hustenintensität ließ schnell nach. Vier Tage nach Behandlungsbeginn war der Bronchitis-Symptomen-Score um die Hälfte reduziert. Auch hier wurde die Verträglichkeit als sehr gut bewertet.

- Quellen: AWMF S3-Leitlinie Nr. 020/003 von 2010; Informationen von Bionorica SE

\section{Differenzierung kann problematisch sein}

\section{Was für ein Typ ist Ihr neu erkannter Diabetes-Patient?}

- Wenn es um Diabetes mellitus geht, ist meist vom häufigsten Typ 2 die Rede. Bedingt durch falsche Ernährung, Bewegungsmangel und demografischen Wandel wird dieser zunehmend zur Volkskrankheit. Aber auch die Prävalenz des Typ-1-Diabetes (T1D) steigt - um ca. $4 \%$ pro Jahr. Prof. Anette-Gabriele Ziegler, München, forderte deshalb eine stärkere Konzentration auf diese seltenere Form der Erkrankung und rief zur vermehrten Aufklärung auf.

Eine Differenzierung zwischen Typ-1und Typ-2-Diabetes ist nicht immer einfach. Generell handelt es sich bei T1D um eine Autoimmunerkrankung, die eine Zerstörung der Betazellen und damit einen zunehmenden Insulinmangel bewirkt, während bei T2D eine Insulinresistenz vorliegt. In der Praxis kann es jedoch auch zu Mischformen, wie dem sogenannten „LA-
DA-(latent insulinpflichtiger Diabetes mellitus im Erwachsenenalter-)Diabetes" kommen. Dieser ist dem Komplex des T1D zuzuordnen, zeichnet sich jedoch durch eine zusätzliche Insulinresistenz aus.

\section{Differenzialdiagnostische Hinweise}

Da das Krankheitsbild bei T1D und T2D oft sehr ähnlich ist, kann bei Verdacht auf T1D oder bei entsprechender Familienanamnese ein Immun-Screening hilfreich sein. Finden sich Antikörper gegen Insulin (IAA), Glutamatdecarboxylase (GADA) oder Thyrosinphosphatase (IA2A), ist ein zukünftiger oder neumanifester T1D wahrscheinlich. Auch die Analyse von Begleiterkrankungen könne aufschlussreich sein, erklärte Ziegler. So sei T1D z.B. mit HashimotoThyreoiditis, Zöliakie oder perniziöser Anämie assoziiert, während T2D vornehmlich mit arterieller Hypertonie, Fettstoffwechselstörungen und Adipositas einhergehe.

Langzeitstudien zeigen über Jahre hinweg stabile $\mathrm{HbA}_{1 c}$-Werte für Typ-1-Diabetiker unter intensivierter konventioneller Insulintherapie (ICT). Die Therapie des „Doppeldiabetes" gestaltet sich schwieriger: Ziegler empfiehlt die Gabe von anfangs $2 x$ $500 \mathrm{mg}$ Metformin zusätzlich zu einer ICT; gleichzeitig könne man gegebenenfalls eine Insulinreduktion von ca. 10\% der Langzeit-Insulinmenge vornehmen. Das Problem dabei ist, dass Metformin bislang ausschließlich zur Therapie des T2D zugelassen ist. Der Einsatz bei T1D ist somit offlabel - ein Umstand, der sich nach Ansicht Zieglers möglichst bald ändern sollte.

- Sonja Kauffmann

Quelle: Kolloquium „DiaLoQ - Im Fokus: Typ 1“, Potsdam (Veranstalter: Berlin-Chemie AG) 